\title{
Preliminary Comparison of the Performance of Thermodynamic Models of the Subsonic Ejector and Turbofan
}

\author{
Vytautas Martinaitis - Dovydas Rimdžius - Juozas Bielskus - Giedrẻ Streckienë* - Violeta Motuzienė \\ Vilnius Gediminas Technical University, Department of Building Energetics, Lithuania
}

Flow-mixing is common in technological processes, and both ejectors and turbofans can produce an interaction between flows with different energy potentials. Ejectors have been extensively used, and their analytical models have been widely presented. To the best of the authors' knowledge, there is a lack of studies exploring the efficiencies of turbofans. The goal of this paper is to compare thermodynamic processes in these devices. Their efficiencies are compared in terms of compression and entrainment ratios. A comprehensive one-dimensional subsonic thermodynamic models of the ejector and turbofan are presented. The quantitative indices are obtained for the same initial conditions expressed as the ratio of differences in enthalpies for ideal compression and ideal expansion. When initial conditions are equal to the numeric value of the combination of the isentropic efficiency of the evaluated components (numerical case 0.16), both devices have the same efficiencies; at lower initial conditions (numerical case 0.10), a turbofan's entrainment ratio is 1.5 times and compression efficiency 1.25 times higher; at higher values of initial conditions (numerical case 0.28 ), the ejector is more efficient. Such distinctive characteristics of turbofans and the nature of their variation may correspond to the specific application areas of technological equipment that require certain flow-mixing parameters.

Keywords: ejector, turbofan, thermodynamic model, flow mixing, efficiency of compression, entrainment ratio

\section{Highlights}

- Thermodynamic processes of gas mixing in the ejector and the turbofan are compared.

- The efficiency of compression and entrainment ratios are compared in subsonic mode.

- When entrainment ratios are low, the ejector is more advantageous than turbofan.

- Turbofan can be applied where a relatively higher passive flow rate is required.

- Isentropic efficiencies are highly dependent on the features of each component.

\section{INTRODUCTION}

Flow-mixing processes are common in the devices of many technological systems. These processes are realized in components such as ducts, nozzles, diffusers, throttling valves, mixing chambers, turbines, compressors, and ventilators. Mixing flows with different parameters in the mixing chamber are usually aimed at the increase of the efficiency and operational abilities of these devices. The presented analysis relates to different flow-mixing devices in which the mixing of two gas flows takes place due to different energy potentials. Such cases are sketched in Fig. 1.

The ejector (Fig. 1a) is a very well-known device, and the roof turbine ventilator (Fig. 1b) has become ubiquitous over the last decade as a device using renewable wind energy for building ventilation. Turbofans - gas mixers (Fig. 1c) are used rarely, although they have been known for a long time as gas burners' mixers. Despite similarities with other mentioned devices, turbofans - engines (Fig. 1d) will not be discussed further because of their complexity and significant differences in scope of operation compared to other above-described mixers.
The roof turbine rotor (Fig. 1b) on the side from which the wind blows operates as turbine, and as a fan from the opposite side. Ejection processes are also happening in it. During the operation of this device, very complex processes occur, which could be why, to the best of the authors' knowledge, there has been no theoretical, thermodynamic model to describe the operation of the roof turbine ventilator to date. In the provided examples, the interaction and mixing between flows that have varying energy potentials are common. The models of classical thermodynamic analysis enable understanding the operation of the technical system; determing the places and causes of the irreversibility of energy conversion processes, as well as comparing the performance of the technical systems that are described in the same way.

This paper focuses on a conceptual comparison of the two shown devices: the ejector (Fig. 1a) and the turbofan-gas mixer (Fig. 1c). The ejector will be used as baseline for such comparison, as ejectors thermodynamic models have strong practical and scientific validation. The turbofan (Fig. 1c) has a separated turbine and fan; therefore, it preliminary comparison requires less debatable assumptions (e.g. in comparison to the roof turbine ventilator (Fig. 1b)) and has a close logical sequence same as ejector. 
Nowadays, the application of ejectors has spread to various thermal engineering facilities. Ejectors are used in various areas: vapor-compression refrigeration systems [1]; in buildings and vehicles ventilation [2], district heating [3], etc. However, recently the application and improvement of ejectors in refrigeration equipment has been at the centre of most studies [4].

A review of various research on the mathematical models of the hydrodynamic and thermodynamic nature of the ejector is provided in [5]. The thermodynamic model of the ejector is developed based on balance equations of mass, momentum and energy with specific interpretations for the ejector or its sections. According to the review [5] in the majority of cases the flows inside the ejector can be treated as steady and one-dimensional. The friction of flows and non-ideal mixing leads to a non-isentropic process, which is evaluated by isentropic efficiencies in the nozzle, mixing and diffuser sections [1] and [6].

The compression efficiency of the ejector [5] and [7] is evaluated in terms of the energy recovered by the secondary flow with respect to the energy available in the primary flow. The above-mentioned ejectors' models [5], characteristics of their properties [1] and [8] are widely validated experimentally or tested in practice.

As was mentioned and shown above (Fig. 1c), a turbofan has a turbine and fan located on a common

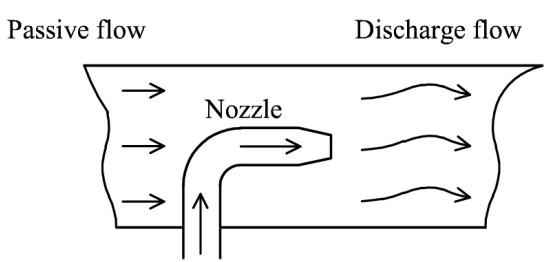

Active flow

(a)

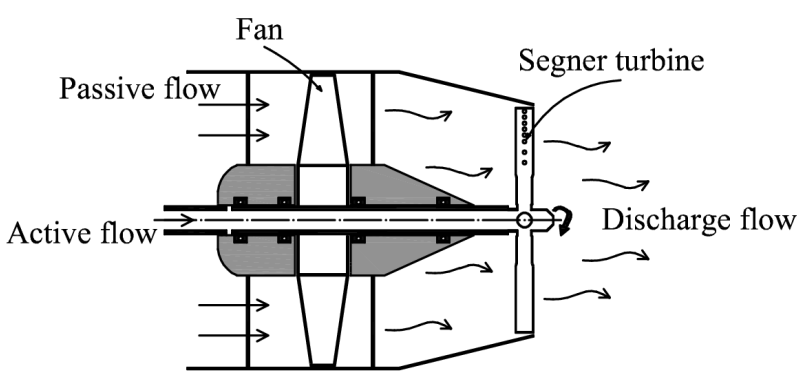

(c) axis. There are application examples in which the renewable energy driven wind turbine is employed to rotate fans to ventilate road tunnels [9]. This type of turbofan is often applied in industrial applications [10] when burning natural gas.

When discussing the turbofans, one should bear in mind the idiosyncrasies of microturbines [11]. These devices were first used in dental medicine [12] before spreading to small-scale energy systems [13] or other micro systems [14]. In the context of the present analysis, it is important to note that the performance indicators of microturbines differ significantly from traditional energy turbines [15].

There are dozens of patents, the majority of which involve gas burners [16] and [17]. Unfortunately, scientific papers rarely focus on such specifics; to the best of authors' knowledge, no thermodynamic models opeartions are described in a manner similar to the way ejectors are described, which hinders the preliminary assessment of their applicability.

The distinctive characteristics of turbofans as well as the nature of their variation may correspond to the specific application areas of the technological equipment (e.g. ventilation, air conditioning, refrigeration), which require certain flow-mixing parameters. These may be relatively new areas, such as personalized ventilation [18], compressed air energy storage [19], or integration in road tunnel ventilation [9] and [20]. When considering the potential

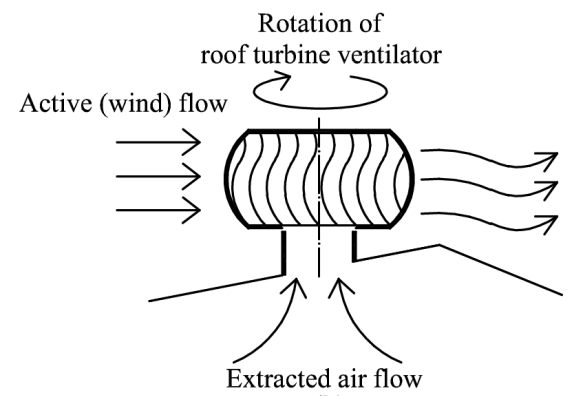

(b)

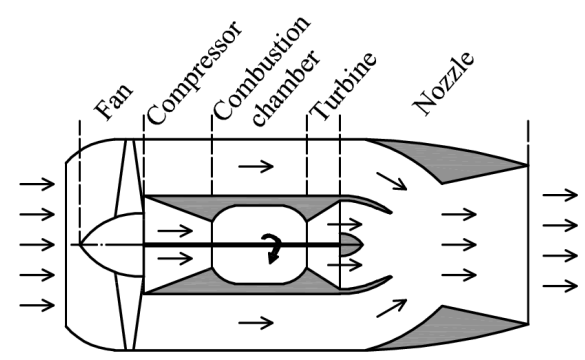

(d)

Fig. 1. Devices, the functionality of which is obtained by using the potential and kinetic energy of flows by mixing them: a) ejector; b) roof turbine ventilator; c) turbofan - gas mixer; d) turbofan - engine 
application of a turbofan, preliminary indications about the basic characteristics, such as the efficiency of compression and entrainment ratios, are required. For these applications, supersonic flow is not typical, so the methodology developed below in the paper is limited to the analysis of subsonic modes.

There is a knowledge gap regarding which turbofan or ejector is superior or inferior to each other. The goal of this paper is to compare thermodynamic processes in the ejector and the turbofan when they have the same initial states of active and passive flows. The path to obtaining a turbofan's characteristics based on the adapted thermodynamic model of the ejector was chosen. The proposed model is compared to well-known, broad-mode and fluid range validated ejector thermodynamic models. The latter have been modified and detailed here to allow symmetric comparison of ejector and turbofan thermodynamic models. It is very important to determine the internal irreversibility (losses) of the processes.

Quantitative indicators required to solve the tasks are defined using analytical, empirical, and numerical models as well as a combination of their equations. The presented preliminary comparison of performance thermodynamic models for the mentioned devices has not been described elswhere in the literature. The present article should be considered as a theoretical or conceptual one. The analytical equations of flow processes and their interpretations found in the textbooks of engineering thermodynamics are chosen for this case and prevail in the article. The authors believe that using/selecting five process irreversibility coefficients for the produced entropy enabled minimizing the empiricism taht common in technical sciences.

This article is structured as follows: Section 1 explains the ideal processes in ejector and turbofan. Section 2 describes in detail the adapted thermodynamic model of the process in the ejector. Section 3 presents the thermodynamic model of the turbofan in parallel with the main accents of the ejector model. The assessment and comparison of the main performance indicators (compression and entrainment ratios as the resulting comparative parameters) of both devices for the case study are presented in Section 4. The conclusions are summarized in Section 5.

\section{IDEAL PROCESSES IN THE EJECTOR AND TURBOFAN}

Simplified schemes of the compared devices with their characteristic parameters are presented: ejector (EJ) in Fig. 2 and turbofan (TF) in Fig. 3. The main components of the EJ depicted in Fig. 2 are: nozzle, mixing chamber and diffuser. The main components of the TF in Fig. 3 are the air turbine and fan.

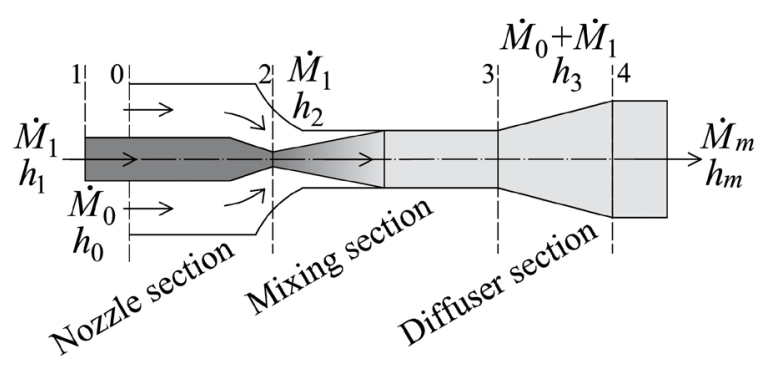

Fig. 2. Simplified scheme of the ejector (the numbers next to the cross-sections correspond with the indices used in equations and diagrams

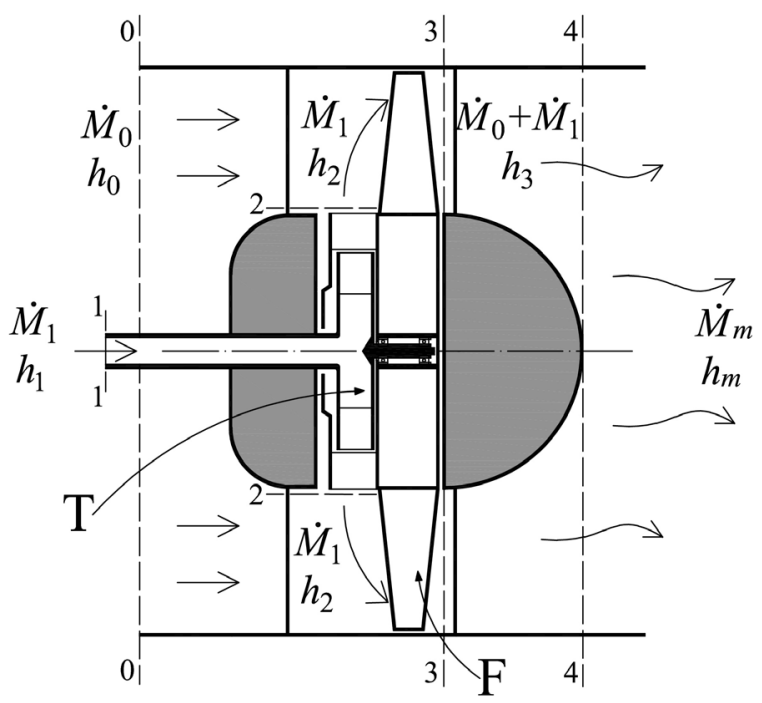

Fig. 3. Simplified scheme of the turbofan: $T$ - turbine, $F$ - fan

The purpose of these devices is to create a mixture that has the required flow mass ratio $\bar{M}=\dot{M}_{0} / \dot{M}_{1}$ and to provide this mixture with pressure $P_{m}$. In both devices, the passive flow of enthalpy $h_{0}$ (or pressure $P_{0}$ ) is induced by the active flow expressed as enthalpy $h_{1}$ (or pressure $P_{1}$ ). These flows are mixed, and the mixture attains the state of enthalpy $h_{m}$ (or pressure $\left.P_{m}\right)$. The following relationship exists between the states that define these processes:

$$
P_{0}<P_{m}<P_{1} \text { or } h_{0}<h_{m}<h_{1} .
$$

The above-mentioned common states for both devices and related ideal gas processes are shown in the $h-s$ diagram (Fig. 4). At first, consider these processes to be ideal when the internal irreversibility in each section is expressed by $\Delta S_{i r}=0$ and let us denote states that correspond with that with the ' index. 
In an ideal case, the initial state of active flow is marked as state 1 and for a passive flow 0 . For the ideal processes, the initial state of the mixture (3') is on the $P_{0}$ isobar that connects states 0 and 2 '. Finally, the resulting state of the mixture is state 4 ', which is on the line that connects states 0 and 1 . The ratio of ideal compression and ideal expansion $\left(h_{4^{\prime}}-h_{3^{\prime}}\right) /\left(h_{1}-h_{2^{\prime}}\right)$ is considered to be an indicator of the identity of the initial conditions of both devices.

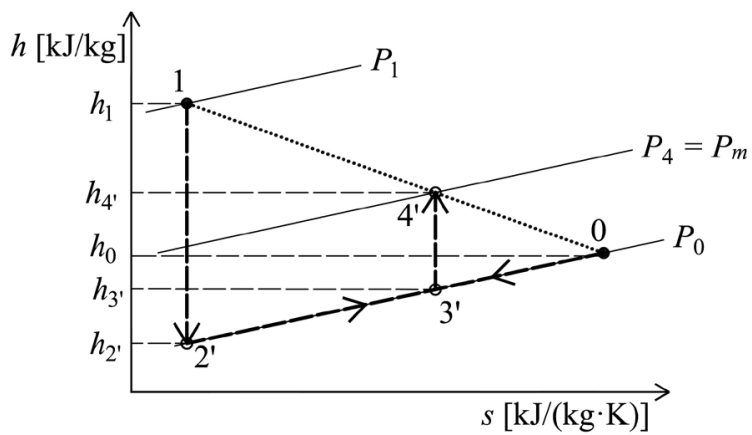

Fig. 4. Ideal mixing processes in ejector and turbofan

In this case the first law of thermodynamics (FLT) and the second law of thermodynamics (SLT) are expressed as follows:

$$
\begin{aligned}
& \dot{M}_{1^{\prime}}^{+} h_{1}+\dot{M}_{0}^{+} h_{0}=\left(\dot{M}_{1^{\prime}}^{-}+\dot{M}_{0}^{-}\right) h_{4^{\prime}}, \\
& \dot{M}_{1^{\prime}}^{+} s_{1}+\dot{M}_{0}^{+} s_{0}=\left(\dot{M}_{1^{\prime}}^{-}+\dot{M}_{0}^{-}\right) s_{4^{\prime}} .
\end{aligned}
$$

The parametric equation resulting from the FLT and SLT enables to determine the specific location of point 4' for the enthalpy and entropy of this mixture:

$$
s_{4^{\prime}}=\frac{\dot{M}_{1} s_{1}+\dot{M}_{0} s_{0}}{\dot{M}_{1^{\prime}}+\dot{M}_{0}} \text { and } h_{4^{\prime}}=\frac{\dot{M}_{1^{\prime}} h_{1}+\dot{M}_{0} h_{0}}{\dot{M}_{1^{\prime}}+\dot{M}_{0}}
$$

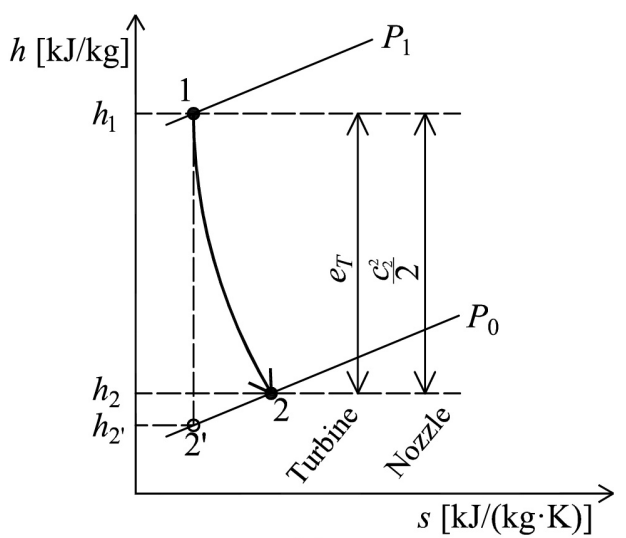

(a)
One of the most important indicators of such a process is the ratio between the flow mass rates, entrainment ratio $\bar{M}_{\text {: }}$ :

$$
\bar{M}_{\mathrm{I}}=\frac{\dot{M}_{0}}{\dot{M}_{1^{\prime}}}=\frac{h_{1}-h_{4^{\prime}}}{h_{4^{\prime}}-h_{0}}=\frac{s_{1}-s_{4^{\prime}}}{s_{4^{\prime}}-s_{0}} .
$$

The location of point $4^{\prime}$ on the diagram allows determining the pressure $P_{m}$ of the mixture in the idealized process.

Another important performance indicator would be the efficiency of compression, which demonstrates the ratio of the achieved result and the accompanying costs:

or

$$
\eta_{m}=\left(\dot{M}_{1^{\prime}}+\dot{M}_{0}\right)\left(h_{4^{\prime}}-h_{3^{\prime}}\right) / \dot{M}_{1^{\prime}}\left(h_{1}-h_{2^{\prime}}\right),
$$

$$
\eta_{m}=\left(1+\bar{M}_{\cdot}\right) \frac{\left(h_{4^{\prime}}-h_{3^{\prime}}\right)}{\left(h_{1}-h_{2^{\prime}}\right)} .
$$

These two indicators together characterize the performance of the device. They must be defined for actual processes, i.e. evaluate the irreversibility of all processes in the devices.

Ideal expansion from 1 to $2^{\prime}$ and compression from $3^{\prime}$ to $4^{\prime}$ and relevant actual processes from 1 to 2 and from 3 to $4_{m}$ for both devices are drawn analogously, as shown in Fig. 5.

Thus, from a graphical point of view, i.e. from the first approach, the processes do not differ in these devices. Both in the nozzle of the EJ and the turbine of the TF the conversion of the state of the active component (that has a higher total energy or enthalpy) to another state takes place. However, there is a difference. Transformations linked to kinetic energy take place in the EJ. In the nozzle of the EJ a flow

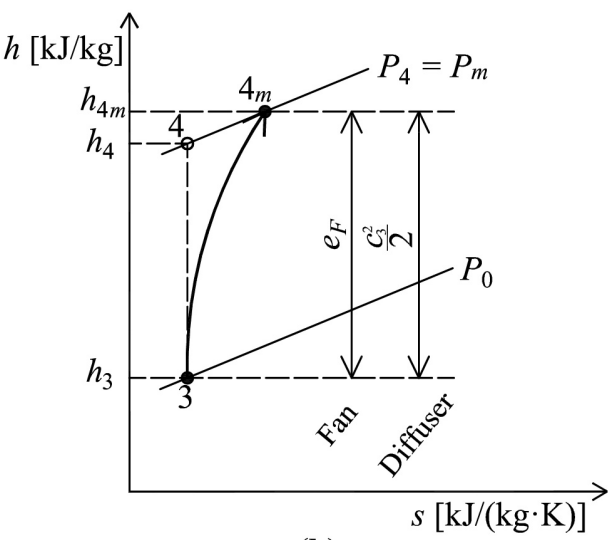

(b)

Fig. 5. Energy conversion processes on the h-s diagram in the components of the ejector and the turbofan: a) the turbine $(T)$ and the nozzle; $b$ ) the compressor or the fan $(F)$ and the diffuser 
process takes place, i.e. the energy of the active component is converted to flow velocity $C_{2}$ (kinetic energy of the flow $C_{2}^{2} / 2$ ). The energy form obtained in the turbine is expressed specific mechanical work of the turbine $e_{T}$. Analogously, only opposite processes occur in the diffuser and the fan. Actual processes properties depend both on the above mentioned inherent differences of the processes, as well as on specific irreversibility properties of their specific components, which will be discussed further, as thermodynamic models for each device are presented.

The processes in this paper are limited to cases in which both fluids are of the same nature and incompressible, and the flow is subsonic. This limitation has no significant effect on the planned essential assessments of the thermodynamic analysis of devices (balance equations and graphical representation) as well as the comparison of processes that take place therein and the efficiency thereof.

The thermodynamic statement, when and how much the device with the energy transformation through mechanical work is more efficient than another device, in which the kinetic energy is directly transferred between the two streams, is the interest of this work. This statement will be proven based on equivalent thermodynamic models for both devices. The proposition of such balanced minuteness models is an important part of this study.

\section{THE THERMODYNAMIC MODEL OF THE PROCESS IN THE EJECTOR}

This work compares and discusses the thermodynamic models of two devices, the ejector and the turbofan, designed to prepare a mixture of the selected mass ratio and state for the two ideal gas subsonic flows. We start with the EJ as a widely-known yet specific device used for flow mixing. Its specific feature is that the energy of the active flow (the flow that has a higher total enthalpy) is used to entrain the passive flow (the flow that has a lower total enthalpy) and to provide the mixture thereof with the momentum required to create the potential and kinetic energy of that mixture and to transport it between the devices in the system.

The structure of thermodynamic ejectors' models designed to assess the processes that take place and related cases have been presented in the introduction. The model is based on balance equations of mass, momentum and energy for the EJ or its sections. In the majority of cases the flows inside the EJ can be treated as steady and one-dimensional.
The specifics of each model usually reflect the objectives and specifics of this paper. The usual sequence is followed in this paper. In order to correctly compare the EJ and TF, certain adjustments have been made.

To compare actual and idealized processes they are drawn on the same $h-s$ diagram (Fig. 6). Dry air when subsonic flow is present has been selected for the case study. The influence of air humidity is not investigated as it is negligible in the context of this research. The initial state is 1 (1.9 bar) for the active flow and 0 (1.01325 bar) for the passive flow. The same pressure $P_{m}$ is to be obtained in the actual process. For this, the mass flow rate from idealized to actual processes should be increased $\dot{M}_{1}>\dot{M}_{1^{\prime}}$. In addition, the state of the resulting mixture in this case on this $P_{m}$ isobar would shift to the right from point 4' to $4_{m}$. We will discuss this transformation step-bystep.

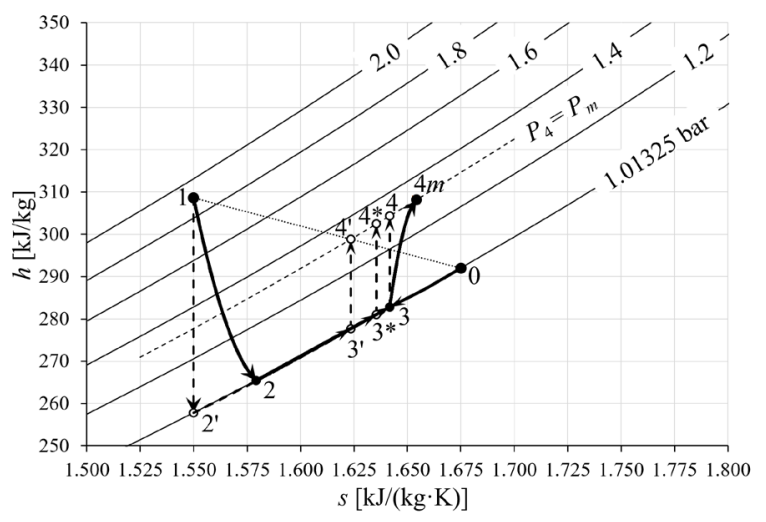

Fig. 6. The depiction of the analyzed processes in the ejector on the $h-s$ diagram

In the actual process, the active flow that flows out of the nozzle expands until it reaches pressure $P_{0}$, state 2 and velocity $C_{2}$, which is expressed as follows:

$$
C_{2}=\sqrt{2\left(h_{1}-h_{2}\right)}=\sqrt{2\left(h_{1}-h_{2^{\prime}}\right) \eta_{N_{S}}},
$$

where $\eta_{N s}=\left(h_{1}-h_{2}\right) /\left(h_{1}-h_{2}\right) \quad-$ the isentropic efficiency of nozzles (see the $h-s$ diagram).

Having thus evaluated the internal irreversibility of the flow in the nozzle, the state of the mixture before the diffuser on the $h-s$ diagram will shift from point $3^{\prime}$ to the right $\Delta s_{N}=\Delta s_{3^{\prime}-3^{*}}$ to point $3^{*}$. Then, after the ideal diffuser we would have the equivalent of point $3^{*}$ - point $4 *$ on the pressure line $P_{m}$. If we consider the velocity equalizing process of both components in the mixing section as isobaric, $P_{2}$ $=P_{3}=P_{0}$, we can assess the internal irreversibility of this process in terms of the produced entropy 
$\Delta s_{M}=\Delta s_{3^{*}-3}$. We have the corresponding enthalpy $h_{3}$ and the isentropic efficiency of mixing chamber $\eta_{M s}$. The authors propose expressing this efficiency, using the process parameters depicted on the $h-s$ diagram, as follows:

$$
\eta_{M s}=\frac{\left(h_{2}-h_{3^{*}}\right)}{\left(h_{2}-h_{3}\right)}
$$

and after the ideal diffuser point 4 on the pressure line $P_{m}$ would be obtained.

When transitioning to the actual diffuser, it is necessary for the velocity $C_{3}$ at the rear of the mixing chamber (i.e., before the diffuser) to become $C_{4} \approx 0$ once the flow reaches the rear of that diffuser on crosssection 4, and that its pressure and enthalpy remain $P_{m}$ and $h_{4 m}$, respectively. Having linked this to the actual process in the diffuser:

$C_{3}=\sqrt{2\left(h_{4 m}-h_{3}\right)}=\sqrt{2 \frac{\left(h_{4}-h_{3}\right)}{\eta_{D s}}}=\sqrt{2 \frac{\left(h_{4^{\prime}}-h_{3^{\prime}}\right)}{\eta_{D s}}}$,

where $\eta_{D s}=\frac{\left(h_{4}-h_{3}\right)}{\left(h_{4 m}-h_{3}\right)}=\frac{\left(h_{4^{\prime}}-h_{3^{\prime}}\right)}{\left(h_{4 m}-h_{3}\right)}$ is the isentropic efficiency of the diffuser. In Eq. (9) and the expression of $\eta_{D s}$ the enthalpy difference was changed in accordance with the equation observed in the process on the $h-s$ diagram, $h_{4}-h_{3}=h_{4^{*}}-h_{3^{*}}=h_{4^{\prime}}-h_{3}$, because isobars $P_{m}$ and $P_{0}$ are almost parallel and the distance in the direction of $s$ in terms of the entire diagram is small.

Velocities $C_{0}, C_{2}$ and $C_{3}$ are related by the process in the mixing chamber, which is denoted by a dashed line in Fig. 2. In quantitative terms, it is defined by the momentum equation using these velocities and corresponding cross-sections:

$$
\begin{aligned}
& P_{2} A_{2}+P_{0} A_{0}+\dot{M}_{1} C_{2}+\dot{M}_{0} C_{0}= \\
& =P_{3} A_{3}+\left(\dot{M}_{0}+\dot{M}_{1}\right) C_{3}+F_{2-3},
\end{aligned}
$$

where $F_{2-3}$ is the force between cross-sections 2 and 3.

When analysing ejectors, it is assumed that the mixing process takes place at a constant pressure $P_{2}=P_{3}=P_{0}$, areas of cross-sections are linked $A_{2}+A_{0}=A_{3}, \quad F_{2-3}=0$ and $C_{0}=0$. To assess these assumptions in quantitative terms, in this paper, we propose using the isentropic efficiency of the mixing chamber and the corresponding produced entropy. Therefore the momentum Eq. (10) can be expressed as $\dot{M}_{1} C_{2} \sqrt{\eta_{M s}}=\left(\dot{M}_{0}+\dot{M}_{1}\right) C_{3}$ and readjusted as follows:

$$
\frac{\dot{M}_{0}}{\dot{M}_{1}}=\sqrt{\eta_{M s}} \frac{C_{2}}{C_{3}}-1 \text {, or } \bar{M}=\sqrt{\eta_{M s}} \frac{C_{2}}{C_{3}}-1 \text {. }
$$

Having used Eq. (7) and Eq. (9), as actual processes take place in the EJ, it follows that:

$$
\bar{M}=\frac{\dot{M}_{0}}{\dot{M}_{1}}=\sqrt{\eta_{N s} \eta_{M s} \eta_{D s} \frac{\left(h_{1}-h_{2^{\prime}}\right)}{\left(h_{4^{\prime}}-h_{3^{\prime}}\right)}}-1 .
$$

Using this equation we can find the actual flow rate of the active fluid $\dot{M}_{1}$ under actual conditions of the process efficiency, evaluated by $\eta_{N s}, \eta_{M s}, \eta_{D s}$. The initial state of the active flow, according to the $h-s$ diagram, is 1 and the initial state of the passive flow (flow to be transported) is 0 and its mass flow rate $\dot{M}_{0}$. When analysing the $h-s$ diagram, these initial conditions can be expressed as follows:

$$
\frac{h_{4^{\prime}}-h_{0}}{h_{1}-h_{0}}=\frac{h_{4^{\prime}}-h_{3^{\prime}}}{h_{1}-h_{2^{\prime}}}=\frac{1}{1+\bar{M}_{1}} \text { or } \bar{M}_{1}=\frac{h_{1}-h_{0}}{h_{4^{\prime}}-h_{0}}-1,
$$

and then the equation that expresses the relationship between the main indicators of the actual and ideal ejectors (i.e., the entrainment ratio and coefficients of isentropic efficiencies of processes), is valid:

$$
\bar{M}=\sqrt{\eta_{N s} \eta_{M s} \eta_{D s}\left(1+\bar{M}_{\cdot}\right)}-1
$$

\section{THE PROCESS IN THE TURBOFAN ALONGSIDE THE PROCESS IN THE EJECTOR}

In the nozzle of the EJ a flow process takes place with energy losses, evaluated as the isentropic efficiency of the nozzle $\eta_{N_{s}}$. In this case there is no mechanical work. A work process takes place in the turbine, with corresponding energy losses, evaluated as the isentropic efficiency of the turbine $\eta_{T}=\left(h_{1}-h_{2}\right) /\left(h_{1}-h_{2}\right)$. Even though this expression is analogous to $\eta_{N s}$, their numeric values differ due to different $\Delta s_{2-2}$, which depends on the distinctive properties of the component (turbine or nozzle).

When presenting the processes in the TF below, the device will be compared with the process in the EJ which was discussed in chapter 2. The work obtained in the turbine and the momentum obtained in the nozzle are used in the next elements of the corresponding device. We have chosen the aggregation of most common losses which occur in the TF. As a result, we used "isentropic efficiency of the turbine" and "isentropic efficiency of the fan". It is hardly possible to estimate all losses that occur in practice. 
In our research, these efficiencies are accepted on the basis of engineering practice and published investigations; the losses in microturbines have been analysed widely in [12], [13], [15] and [16].

The specific mechanical work of the turbine is $e_{T}$ or power $\dot{E}_{T}=e_{T} \dot{M}_{1}$, while the internal irreversibility of this process is evaluated as the isentropic efficiency of the turbine $\eta_{T}$. This power is transmitted to the fan installed on the same shaft without any energy losses. As in any fan, the change of state of the flow that is being mixed $\dot{M}_{1}+\dot{M}_{0}$ takes place here, which is evident as an increase of enthalpy. The internal irreversibility of this process, including mixing and the restoration of pressure in the diffuser that is assigned to the fan, is evaluated as the isentropic efficiency of the fan $\eta_{T}$. In a more detailed analysis it can be divided into impeller, mixing and diffuser losses $\eta_{F}=\eta_{F I} \eta_{F M} \eta_{F D}$.

In quantitative terms, the process in the TF is expressed using the mechanical work of components, which is expressed by the power balance equation:

$$
e_{T} \dot{M}_{1}=e_{F}\left(\dot{M}_{1}+\dot{M}_{0}\right) \text {. }
$$

Both compression processes on $h-s$ diagrams (Figs. 5 and 7) are depicted using process lines with the same indices. Fig. 7 is a simplified version of Fig. 6 . The main processes on the $h-s$ diagram for the EJ and the TF are depicted in an analogous manner.

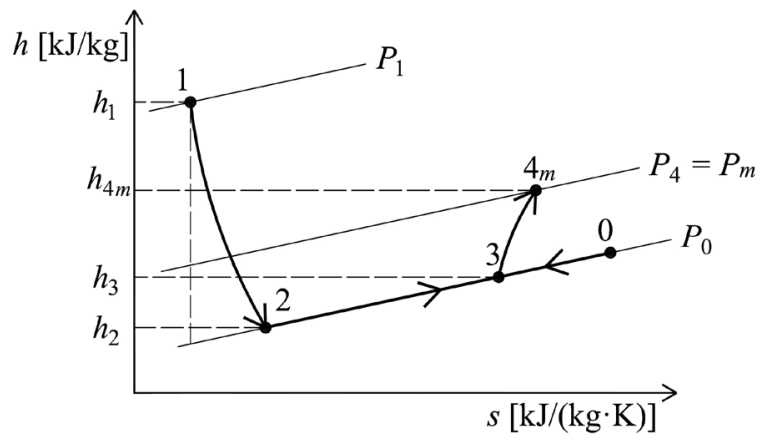

Fig. 7. The processes of the change of state of components that are being mixed in the EJ and the TF

Eqs. (11) and (15) serve as the basis of the dependence on the entrainment ratio for each of the compared devices:

and

$$
\bar{M}_{E J}=\frac{C_{2}}{C_{3}}-1,
$$

$$
\bar{M}_{T F}=\frac{e_{T}}{e_{F}}-1 \text {. }
$$

In general, the velocity of the flow that leaves the nozzle at the defined difference of enthalpies and the isentropic efficiency of the nozzle (the ratio of the difference of actual and isentropic kinetic energies) $\eta_{N s}$ is expressed as follows:

$$
C=\sqrt{2\left(h_{1}-h_{2}\right) \eta_{N s}} .
$$

The specific work in the turbine is calculated likewise:

$$
e=\left(h_{1}-h_{2}\right) \eta_{T} .
$$

Then Eqs. (16a) and (16b) can be rewritten as:

$$
\bar{M}_{E J}=\sqrt{\eta_{N s} \eta_{M s} \eta_{D s} \frac{\left(h_{1}-h_{2^{\prime}}\right)}{\left(h_{4^{\prime}}-h_{3^{\prime}}\right)}}-1,
$$

and

$$
\bar{M}_{T F}=\eta_{T} \eta_{F I} \eta_{F M} \eta_{F D} \frac{\left(h_{1}-h_{2^{\prime}}\right)}{\left(h_{4^{\prime}}-h_{3^{\prime}}\right)}-1 .
$$

The efficiency of compression, based on Eq. (6) for actual process:

$$
\eta_{m}=\left(M_{1}+M_{0}\right)\left(h_{4}-h_{3}\right) / M_{1}\left(h_{1}-h_{2^{\prime}}\right),
$$

or

$$
\eta_{m}=(1+\bar{M}) \frac{\left(h_{4}-h_{3}\right)}{\left(h_{1}-h_{2^{\prime}}\right)} \approx(1+\bar{M}) \frac{\left(h_{4^{\prime}}-h_{3^{\prime}}\right)}{\left(h_{1}-h_{2^{\prime}}\right)}
$$

The enthalpy difference could be changed in accordance with the equation observed in the process on the $h-s$ diagram, $h_{4}-h_{3}=h_{4^{\prime}}-h_{3^{\prime}}$, because isobars $P_{m}$ and $P_{0}$ are almost parallel and the distance in the direction of $s$ in terms of the entire diagram is small.

On the basis of Eqs. (19) and (20) the expressions of the efficiency of the EJ and TF are obtained:

and

$$
\eta_{E J}=\sqrt{\eta_{N S} \eta_{M s} \eta_{D s} \frac{\left(h_{1}-h_{2^{\prime}}\right)}{\left(h_{4^{\prime}}-h_{3^{\prime}}\right)}} \cdot \frac{\left(h_{4}-h_{3}\right)}{\left(h_{1}-h_{2^{\prime}}\right)},
$$

$$
\eta_{T F}=\eta_{T} \eta_{F I} \eta_{F M} \eta_{F D} \frac{\left(h_{1}-h_{2^{\prime}}\right)}{\left(h_{4^{\prime}}-h_{3^{\prime}}\right)} \cdot \frac{\left(h_{4}-h_{3}\right)}{\left(h_{1}-h_{2^{\prime}}\right)} \text {. }
$$

Having applied the previous assumption that $h_{4 *}-h_{3^{*}}=h_{4}-h_{3}=h_{4^{\prime}}-h_{3^{\prime}}$ :

and

$$
\eta_{E J}=\sqrt{\eta_{N s} \eta_{M s} \eta_{D s} \frac{\left(h_{4^{\prime}}-h_{3^{\prime}}\right)}{\left(h_{1}-h_{2^{\prime}}\right)}}
$$

$$
\eta_{T F}=\eta_{T} \eta_{F I} \eta_{F M} \eta_{F D} \frac{\left(h_{1}-h_{2^{\prime}}\right)}{\left(h_{4^{\prime}}-h_{3^{\prime}}\right)} \cdot \frac{\left(h_{4}-h_{3}\right)}{\left(h_{1}-h_{2^{\prime}}\right)} \text {. }
$$

When comparing the efficiencies of these two devices it follows that: 


$$
\frac{\eta_{T F}}{\eta_{E J}}=\frac{\eta_{T} \eta_{F I} \eta_{F M} \eta_{F D}}{\sqrt{\eta_{N s} \eta_{M s} \eta_{D s} \frac{\left(h_{4^{\prime}}-h_{3^{\prime}}\right)}{\left(h_{1}-h_{2^{\prime}}\right)}}},
$$

and the entrainment ratio proportion thereof, with the same main starting thermodynamic parameters $\left(h_{1}-h_{2^{\prime}}\right) /\left(h_{4^{\prime}}-h_{3^{\prime}}\right)$ :

$$
\frac{\bar{M}_{T F}}{\bar{M}_{E J}}=\frac{\eta_{T} \eta_{F I} \eta_{F M} \eta_{F D} \frac{\left(h_{1}-h_{2^{\prime}}\right)}{\left(h_{4^{\prime}}-h_{3^{\prime}}\right)}-1}{\sqrt{\eta_{N s} \eta_{M s} \eta_{D s} \frac{\left(h_{1}-h_{2^{\prime}}\right)}{\left(h_{4^{\prime}}-h_{3^{\prime}}\right)}-1}} .
$$

\section{RESULTS OF THE THERMODYNAMIC ANALYSIS}

\subsection{Processes that The Place in the Ejector}

The numeric analysis of the presented model under the initial conditions listed in Table 1 (dry air and subsonic flow) is presented below.

Table 1. Initial air state parameters for the numerical examples

\begin{tabular}{cccc}
\hline & $h[\mathrm{~kJ} / \mathrm{kg}]$ & $s[\mathrm{~kJ} /(\mathrm{kg} \cdot \mathrm{K})]$ & $P[\mathrm{~Pa}]$ \\
\hline 1 & 308.6 & 1.55 & 190000 \\
\hline 0 & 292.0 & 1.675 & 101325 \\
\hline $2^{\prime}$ & 257.8 & 1.55 & 101325 \\
\hline
\end{tabular}

The $h-s$ diagram in Fig. 8 and Table 2 present the results of three numeric examples of the analytical explanation provided above. These cases correspond with the structure of the detailed diagram depicted in Fig. 6, where the numeric case of $M_{0} / M_{1}=1.33$ is shown. For the purpose of an equivalent comparison of the essential results, identical isentropic efficiencies $\eta_{N_{s}}, \eta_{M s}, \eta_{D s}$ were selected for all cases $(0.85 ; 0.90$; 0.85 , respectively [9]).

In Figs. 5 to 8, closed dots depict the states of the actual process, while open dots are used to convey the concept of thermodynamic models (see Fig. 6).

It should once again be noted that this paper presents a case in which the pressure $P_{m}$ and mass flow rate $\dot{M}_{0}$ of the mixture obtained in the ideal process following the proportions of the component should be preserved when transitioning to the actual process. For this purpose, the mass flow rate of the active fluid is increased in terms of the ideal case, $\dot{M}_{1}>\dot{M}_{1}$. As the $\dot{M}_{0}$ component relatively increases, $P_{m}$ moves towards the pressure line of $\dot{M}_{0}(1.01325$ bar $)$.

The enthalpy of the mixture decreases while the entropy increases, in turn increasing the internal irreversibility of the process. The relationship between Eqs. (13) and (14) shows that this mass flow rate has to be increased. Compared with the ideal process for each case, $M_{0} / M_{1}=2.5 ; 5 ; 9$, the mass flow rate of the active flow has to be increased almost five times while the efficiency of compression is nearly halved. This is depicted in Fig. 9.

Other cases are possible; for example, the aim to preserve the initial ratio of mass flow rates $\bar{M}$, i.e., they should remain the same in both ideal and actual cases. Then the pressure of the mixture would be

\begin{tabular}{|c|c|c|c|c|c|c|c|c|c|}
\hline & \multicolumn{9}{|c|}{ Numeric cases in accordance with Eq. (18) } \\
\hline & \multicolumn{3}{|c|}{$M_{0} / M_{1^{\prime}}=2.5$} & \multicolumn{3}{|c|}{$M_{0} / M_{1^{\prime}}=5$} & \multicolumn{3}{|c|}{$M_{0} / M_{1^{\prime}}=9$} \\
\hline & $\eta_{N s}$ & $\eta_{M s}$ & $\eta_{D s}$ & $\eta_{N_{S}}$ & $\eta_{M s}$ & $\eta_{D s}$ & $\eta_{N s}$ & $\eta_{M s}$ & $\eta_{D s}$ \\
\hline & 0.85 & 0.9 & 0.85 & 0.85 & 0.9 & 0.85 & 0.85 & 0.9 & 0.85 \\
\hline & \multicolumn{9}{|c|}{ State parameters typical of the process } \\
\hline & $h[\mathrm{~kJ} / \mathrm{kg}]$ & $s[\mathrm{~kJ} /(\mathrm{kg} \cdot \mathrm{K})]$ & $P[\mathrm{~Pa}]$ & $h[\mathrm{~kJ} / \mathrm{kg}]$ & $s[\mathrm{~kJ} /(\mathrm{kg} \cdot \mathrm{K})]$ & $P[\mathrm{~Pa}]$ & $h[\mathrm{~kJ} / \mathrm{kg}]$ & $s[\mathrm{~kJ} /(\mathrm{kg} \cdot \mathrm{K})]$ & $P[\mathrm{~Pa}]$ \\
\hline 2 & 265.4 & 1.579 & 101325 & 265.4 & 1.570 & 101325 & 265.4 & 1.579 & 101325 \\
\hline $3^{\prime}$ & 282.2 & 1.639 & 101325 & 286.3 & 1.654 & 101325 & 288.6 & 1.663 & 101325 \\
\hline $4^{\prime}$ & 296.8 & 1.639 & 121331 & 294.8 & 1.654 & 113029 & 293.7 & 1.663 & 108696 \\
\hline $3^{*}$ & 284.6 & 1.648 & 101325 & 287.7 & 1.659 & 101325 & 289.4 & 1.665 & 101325 \\
\hline $4^{*}$ & 299.2 & 1.648 & 121331 & 296.2 & 1.659 & 113029 & 294.5 & 1.665 & 108696 \\
\hline 3 & 286.7 & 1.655 & 101325 & 290.2 & 1.668 & 101325 & 292.1 & 1.675 & 101325 \\
\hline 4 & 301.5 & 1.655 & 121331 & 298.8 & 1.668 & 113029 & 297.2 & 1.675 & 108696 \\
\hline \multirow[t]{5}{*}{$4 m$} & 304.1 & 1.664 & 121331 & 300.3 & 1.673 & 113029 & 298.2 & 1.678 & 108696 \\
\hline & \multicolumn{9}{|c|}{ Main results } \\
\hline & $M_{0} / M_{1}$ & $M_{0} / M_{1^{\prime}}$ & $\eta_{m}$ & $M_{0} / M_{1}$ & $M_{0} / M_{1}$ & $\eta_{m}$ & $M_{0} / M_{1}$ & $M_{0} / M_{1^{\prime}}$ & $\eta_{m}$ \\
\hline & Eq. (12) & Eq. (13)/ Eq. (14) & Eq. (20) & Eq. (12) & Eq. (13)/ Eq. (14) & Eq. (20) & Eq. (12) & Eq. (13)/ Eq. (14) & Eq. (20) \\
\hline & 0.509 & 4.916 & 0.438 & 0.975 & 5.128 & 0.334 & 1.550 & 5.806 & 0.258 \\
\hline
\end{tabular}

Table 2. The results for three cases when subsonic flows of air are mixed in the ejector 

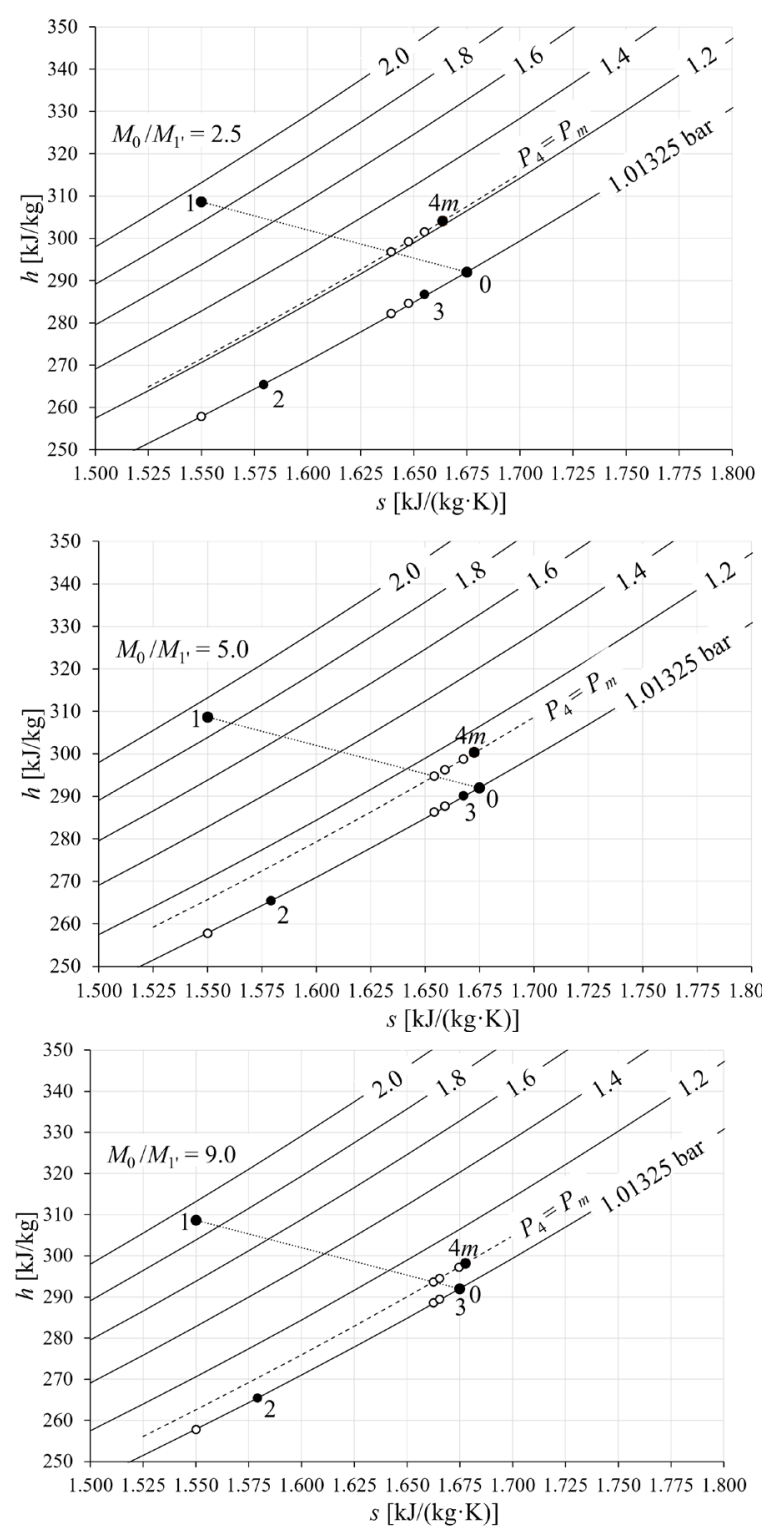

Fig. 8. The depiction of numeric examples of the process in the ejector on the $h$-s diagram: a) when $M_{0} / M_{1},=2.5$; b) when $M_{0} / M_{1}=$ 5; c) when $M_{0} / M_{1}=9$. Other data correspond with Tables 1 and 2

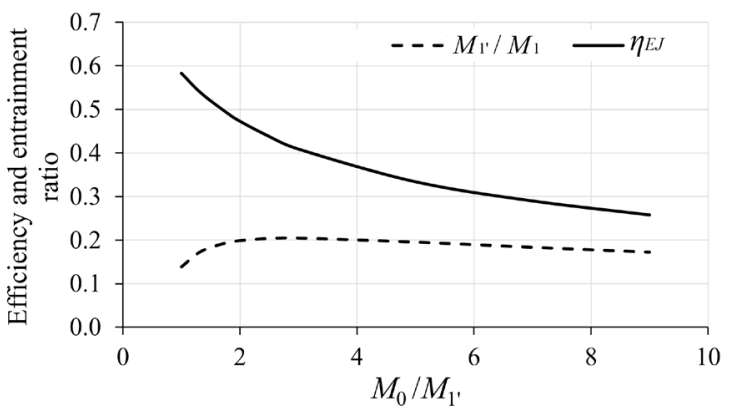

Fig. 9. Variation in actual active mass flow rate and the efficiency of compression of the ejector subject to the selected mass flow ratio in the idealized process lower than $P_{m}$, significantly reducing the efficiency. The results of the comparison of the TF and EJ are presented below.

\subsection{Assessment of the Main Performance Indicators of the Ejector and the Turbofan}

Fig. 10 shows the results of the comparison of the most important performance characteristics for the processes that take place in the EJ and TF, obtained using Eqs. (22) to (24). All those dimensionless or ratio-based characteristics are given depending on the previously mentioned initial conditions $\left(h_{4},-h_{3^{\prime}}\right) /\left(h_{1}-h_{2^{\prime}}\right)$. These conditions are unambiguously related to the entrainment ratio of the ideal EJ, expressed by Eqs. (13) or (5). Fig. 10 shows the numerical link between the following initial conditions and three cases of $M_{0} / M_{1}$, (see Table 2) for the EJ: 2.5, 5.0 and 9.0.

The figure shows that according to the equations obtained during the analysis, the devices compared here have different sensitivities to the initial conditions. When both Eqs. (23) and (24) are equal to 1 (see also 1 in the ordinate axis), the performance indicators for the same initial flow conditions for both devices are the same. From here towards lower values of $\left(h_{4^{\prime}}-h_{3}{ }^{\prime}\right) /\left(h_{1}-h_{2^{\prime}}\right)$ (on the left side of the diagram) the TF has an advantage over the EJ. As shown in the figure, the actual efficiency of compression of the EJ at that location is about $33 \%$ (Eq. 21a) and the entrainment ratio is almost 1 (Eq. 19a).

The intersection of the indicators depicted in the diagram (coloured dots) depends on the numeric values of the combination of isentropic efficiencies $\eta_{T} \eta_{F}$ and $\eta_{N s} \eta_{M s} \eta_{D s}$. The above are commonly assumed to be (point and lines - green): $\eta_{T}=0.5 ; \eta_{F I}=0.8 ; \eta_{F M}=0.9$; $\eta_{F D}=0.9$. Thus, $\eta_{F}=0.8 \times 0.9 \times 0.9=0.65$. For EJ $\eta_{N s}=0.85, \eta_{M s}=0.90, \eta_{D s}=0.85$.

This competitiveness boundary of the compared devices is determined analytically as $\left(\eta_{T} \eta_{F}\right)^{2} /\left(\eta_{N s} \eta_{M s} \eta_{D s}\right)$, which would be an indicator comparing the degree of irreversibility of the processes in these devices, for which no flow state parameters are required. In this case study it equals 0.16 from $(0.5 \times 0.65)^{2} /(0.85 \times 0.90 \times 0.85)$ while the corresponding $\dot{M}_{0} / \dot{M}_{1}$, equals 5.07 .

When we increase the isentropic efficiency of EJ components, we will have the case $<0.16$ (the point of intersection of the comparative parameters will move to the left, see Fig. 10). When we increase the isentropic efficiency of microturbine or fan, we will have a case $>0.16$. 


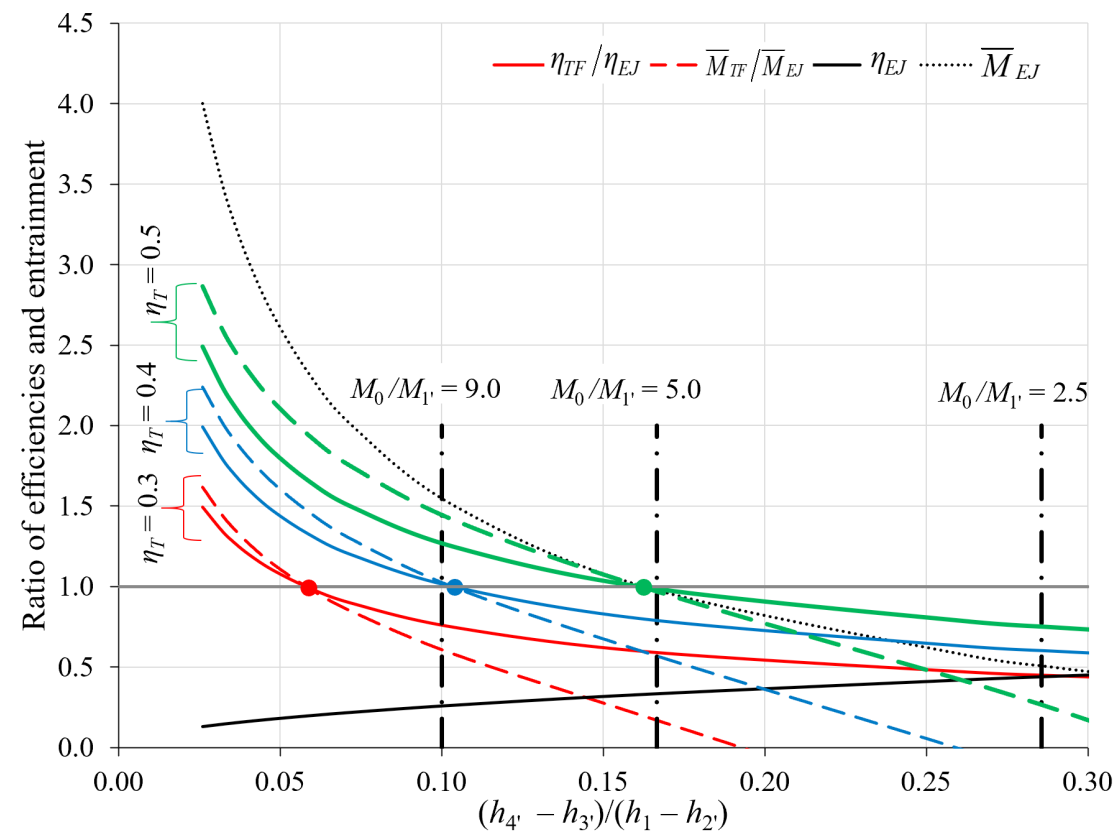

Fig. 10. The comparison of the main performance indicators of the ejector and turbofan under the same starting conditions $\left(h_{4^{\prime}}-h_{3^{\prime}}\right) /\left(h_{1}-h_{2}\right)$, Eq. (13)

These isentropic efficiencies are highly dependent on the size and other features of each evaluated component. While in a wide range $\eta_{N s} \eta_{M s} \eta_{D s}$ are rather stable and well-researched, when it comes to small devices (micro-turbines with partial admission and micro-fans or micro-compressors) $\eta_{T} \eta_{F}$ can move quite far from 1.

This is demonstrated by additional sensitivity analysis by changing the efficiency of the microturbine, which is the parameter whose value has the least certainty at this stage of the research. The ejector's isentropic efficiencies are assumed as constant (see also Table 2) As already mentioned, in the case of Fig. 10, isentropic efficiency of microturbine is $\eta_{T}=0.5$. In the case of $\eta_{T}=0.40$, it is understood that the advantages of TF occur at the EJ entrainment ratio values higher than 1 . These would be the cases to the left of $\bar{M}_{E J} \approx 1.5$ (point and blue lines). In the case of $\eta_{T}=0.30$ of $\mathrm{TF}$, the benefits appear for cases already to the left of $\bar{M}_{E J} \approx 2.3$ (point and red lines). The developed analytical model and this fragment of the sensitivity analysis show that particular attention must be paid at the microturbine's design in the design of TF.

The advantage of the TF over the EJ increases as the entrainment ratio on the basis of mass $\bar{M}=\dot{M}_{0} / \dot{M}_{1}$ increases. This means that based on the $h-s$ diagram, when the initial and resulting state parameters are the same, the TF with the selected active flow rate will allow a higher amount of the passive flow to be pumped.

Due to the higher efficiency of compression and the accompanying entrainment ratios the TF could be relevant in terms of gas burners, roof turbofans and other specific devices. Natural gas burners that use full mixing require $\bar{M} \approx 18$. When entrainment ratios are low, the EJ has an advantage in terms of these indicators. The processes that take place in the TF and EJ are rather simplified in this paper, which allows the authors to easily demonstrate or emphasize that in order to achieve higher entrainment ratios, converting the available energy to mechanical work enables the possibility of achieving better performance when compared with conversion to kinetic energy.

More thorough experimental research is required to determine the values of $\bar{M}$ and the absolute values of $\dot{M}_{1}$ and $\dot{M}_{0}$ at which it is efficient to use the TF. In addition to this, this device has moving parts, making its manufacturing process more complicated.

\section{CONCLUSIONS}

In this paper, the authors have analysed the thermodynamic processes in the ejector and the 
turbofan when they have the same initial states of active and passive flows. This analysis is based on the parallel comparison of two thermodynamic models: one of them is created for turbofan, and one is adapted from the classical ejector. The characteristics that demonstrate their efficiency - the efficiency of compression and entrainment ratios - were defined and compared at the subsonic flow mode conditions. The following conclusions were made:

1. The main cause of the resulting differences is the following. The mixing process in the EJ is realized via the interaction of kinetic energies that is expressed by the momentum balance equation. In the TF this occurs by transforming the energy of the active flow into the turbine work transferred to the fan, which conveys the passive flow. The process is defined by the work balance equations.

2. The case of numerical examples for the comparison of thermodynamic processes in the EJ and the TF when they have same initial states of active and passive flows $\left(h_{4^{\prime}}, h_{3^{\prime}}\right) /\left(h_{1}-h_{2^{\prime}}\right)$ shows that the TF has an advantage over the EJ at lower values of these starting conditions $(<0.161)$. The advantage of the TF over the EJ increases as the entrainment ratio on the basis of mass increases $\bar{M}=\dot{M}_{0} / \dot{M}_{1}$. In this case the turbofan could be relevant in terms of gas burners, roof turbofans or other specific devices for which a relatively higher passive flow rate is required.

3. The boundary between the advantage of the TF over the EJ on the basis of efficiency of compression and entrainment ratio indicators for the same initial flow conditions is determined analytically. These cases correspond to $\left(\left(\eta_{T} \eta_{F}\right)^{2}\right) /\left(\eta_{N s} \eta_{M s} \eta_{D s}\right)$. It is 0.16 in the case of numerical examples and depends on the combination of isentropic efficiency of the components of both devices. In order to determine these specifics for the $\mathrm{TF}$, a thorough experimental study is required, especially if a microturbine with partial admission is used.

The numerical results obtained comparing the models discussed in the paper are limited to air-toair flows mixing at the subsonic regime. Considering similarities to EJ, turbines and ventilators, the presented thermodynamic model could serve as a basis for the creation of the theoretical model for roof turbine ventilators. The developed model could also serve as a basis for its development into supersonic analysis.

\section{ACKNOWLEDGEMENTS}

This project has received funding from the European Regional Development Fund project No 01.2.2-LMT-K-718-01-0016 under a grant agreement with the Research Council of Lithuania (LMTLT).

\section{NOMENCLATURE}

$A$ cross-section area, $\left[\mathrm{m}^{2}\right]$

$C$ velocity, $[\mathrm{m} / \mathrm{s}]$

$\dot{E}$ mechanical power, $[\mathrm{kW}]$

e specific mechanical work, $[\mathrm{kJ} / \mathrm{kg}]$

$F_{2-3}$ power between cross-sections 2 and 3, [N]

$h$. enthalpy, $[\mathrm{kJ} / \mathrm{kg}]$

$\dot{M}$ mass flow rate, $[\mathrm{kg} / \mathrm{s}]$

$\bar{M}$ entrainment ratio (ratio of mass flow rates), [-]

$P$ pressure, $[\mathrm{Pa}]$

$S$ entropy, $[\mathrm{kJ} / \mathrm{K}]$

$s \quad$ entropy, $[\mathrm{kJ} /(\mathrm{kg} \cdot \mathrm{K})]$

$\eta \quad$ efficiency, [-]

$\eta_{D s}$ the isentropic efficiency of diffuser, [-]

$\eta_{F}$ the isentropic efficiency of fan, [-]

$\eta_{F D}$ the efficiency of fan diffuser, [-]

$\eta_{F I}$ the efficiency of fan impeller, [-]

$\eta_{F M}$ the efficiency of mixing in fan, [-]

$\eta_{M s}$ the isentropic efficiency of mixing chamber, [-]

$\eta_{M s}$ the isentropic efficiency of nozzles, [-]

$\eta_{T}$ the isentropic efficiency of turbine, [-]

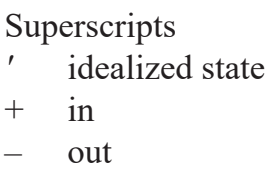

Subscripts

0 to 4 air flow states at the cross-section (according to Fig. 2)

$D$ diffuser

EJ ejector

$F$ fan

ir irreversible

$M$ mixing chamber

$m$ mixture

$N$ nozzle

$T$ turbine

TF turbofan

\section{REFERENCES}

[1] Ma, Z., Bao, H., Roskilly, A.P. (2017). Thermodynamic modelling and parameter determination of ejector for ejection refrigeration systems. International Journal of Refrigeration, vol. 75 , p. 117-128, D0l:10.1016/j.ijrefrig.2016.12.005. 
[2] Ray, S.D., Glicksman, L.R. (2013). Increased natural ventilation flow rates through ventilation shafts. International Journal of Ventilation, vol. 12, p. 195-210, Dol:10.1080/1473 3315.2013.11684016.

[3] Berisha, X., Meha, D. (2018). Modelling of direct connection of heat consumers to district heating with ejectors. International Journal on Advanced Science, Engineering and Information Technology, vol. 8, no. 1, p. 219-226, D0l:10.18517/ ijaseit.8.1.3246.

[4] Besagni, G., Mereu, R., Inzoli, F. (2016). Ejector refrigeration: A comprehensive review. Renewable and Sustainable Energy Reviews, vol. 52, p. 373-407, D0l:10.1016/j.rser.2015.08.059.

[5] He, S., Li, Y., Wang, R.Z. (2009). Progress of mathematical modeling on ejectors. Renewable and Sustainable Energy Reviews, vol. 13, no. 18, p. 1760-1780, D0l:10.1016/j. rser.2008.09.032.

[6] Antonio, Y.M., Périlhon, C., Descombes, G., Chacoux, C. (2012). Thermodynamic modelling of an ejector with compressible flow by a one-dimensional approach. Entropy, vol. 14, no. 4, p. 599-613, D0l:10.3390/e14040599.

[7] Banasiak, K., Palacz, H., Hafner, A., Buliński, Z., Smołka, J., Nowak, A.J., Fic, A. (2014). A CFD-based investigation of the energy performance of two-phase R744 ejectors to recover the expansion work in refrigeration systems: An irreversibility analysis. International Journal of Refrigeration, vol. 40, p. 328-337, D0I:10.1016/j.ijrefrig.2013.12.002.

[8] Liu, F. (2014). Review on Ejector Efficiencies in Various Ejector Systems. Proceedings of the $15^{\text {th }}$ International Refrigeration and Air Conditioning Conference, p. 1-10.

[9] Wang. Z., Wu, Y., Lu, S., Meng, X., Zhang, J. (2019). A study on model experiment and aerodynamic match of wind energy fan (WEF). Sustainable Cities and Society, vol. 49, art. ID. 101618, DOI:10.1016/j.scs.2019.101618.

[10] GazTechSnab Turbojet Gas Burner (Горелка газовая турбореактивная) from: http://www.gaztehsnab.ru/products/ gas-on-fire/gas-on-fire_13.html, accessed on 2019-10-10. (in Russian)

[11] Capata, R., Saracchini, M. (2018). Experimental campaign tests on ultra micro gas turbines, fuel supply comparison and optimization. Energies, vol. 11, no. 4, art. ID 799. DOI:10.3390/en11040799.

[12] Juraeva, M., Park, B.H., Ryu, K.J., Song, D.J. (2017). Designing high-speed dental air-turbine handpiece by using a computational approach. International Journal of Precision Engineering and Manufacturing, vol. 18, p. 1403-1407, DOI:10.1007/s12541-017-0167-4.

[13] Khamis, A., Badarudin, Z.M., Ahmad, A., Rahman, A.A., Bakar, N.A. (2011). Development of mini scale compressed air energy storage system. Proceedings of the IEEE 1st Conference on Clean Energy and Technology, p. 151-156, Dol:10.1109/ CET.2011.6041477.

[14] Sun, S.C., Shi, G.C. (2011). Design, simulation and fabrication of an air-driven microturbomachine. Key Engineering Materials, vol. 483, p. 611-615, D0l:10.4028/www.scientific. net/KEM.483.611.

[15] Capata, R., Sciubba, E. (2015). Experimental fitting of the re-scaled Balje maps for low-reynolds radial turbomachinery. Energies, vol. 8, no. 8, p. 7986-8000, Dol:10.3390/ en8087986.

[16] Adinskov. B.P. (1969). Turbo gas burner. Patent No. 262304 (USSR), Russian Patent Office, Moscow.

[17] Achmedov, P.B., Rashidov, F.K. (1970). Turbine gas burner. Patent No. 262304 (USSR), Russian Patent Office, Moscow.

[18] Dalewski, M., Melikov, A.K., Vesuly, M. (2014). Performance of ductless personalized ventilation in conjunction with displacement ventilation: Physical environment and human response. Building and Environment, vol. 81, p. 354-364. D0l:10.1016/j.buildenv.2014.07.011.

[19] Tallini, A., Vallati, A., Cedola, L. (2015). Applications of micro-CAES systems: Energy and economic analysis. Energy Procedia, vol. 82, p. 797-804, D0l:10.1016/j. egypro.2015.11.815.

[20] Muhasilovic, M., Duhovnik, J. (2012). CFD-based investigation of the response of mechanical ventilation in the case of tunnelfire. Strojniški vestnik - Journal of Mechanical Engineering, vol. 58, no 3, p. 183-190, D0l:10.5545/sv-jme.2009.091. 